\title{
CORRECTIONS TO MY PAPER “ON KRULL'S CONJECTURE CONCERNING VALUATION RINGS”
}

\author{
MASAYOSHI NAGATA
}

The proof of Theorem 1 in the paper "On Krull's conjecture concerning valuation rings" (vol. 4 (1952) of this journal) is not correct." We want to give here a corrected proof of the theorem: From p. $30, l .14$ to p. $31, l .7$ should be changed as follows.

Further we observe that if $w(a-b)>2 \alpha$, then $(x+a) /(x+b)$ is unit in $\mathfrak{D}$. Hence we may assume that $w\left(a_{i}-b_{j}\right)<2 \alpha$ for any $(i, j)$.

Next, we will show two lemmas concerning the valuations $w_{\lambda}$ and $w_{e}$ :

Lemma A. Set $d=\Pi_{1}^{n^{\prime}}\left(x+a_{i}\right) / \Pi_{1}^{m^{\prime}}\left(x+b_{j}\right)$ and assume that $w\left(a_{i}\right)=w\left(b_{j}\right)$ $=\sigma(a<\sigma<2 \alpha)$ for any $i$ and $j$. Let $e$ be any element of $K$ such that $w(e)$ $=\sigma$. Then either $w_{e}(d) \geqq w_{\sigma}(d)$ or there exists one $b_{j}$ such that $w_{e}(d) \geqslant w_{b_{j}}(d)$.

Proof. We may use the induction argument on $m^{\prime}+n^{\prime}$. Obviously $w_{e}(x$ $\left.+a_{i}\right)=\min \left(w\left(a_{i}-e\right), 2 \alpha\right), w_{e}\left(x+b_{j}\right)=\min \left(w\left(b_{j}-e\right), 2 \alpha\right):$ Let $\sigma^{\prime}$ be the maximum of these values. We renumber $a_{i}$ and $b_{j}$ so that $w_{e}\left(x+a_{i}\right)=w_{e}(x$ $\left.+b_{j}\right)=\sigma^{\prime}$ if and only if $i \leqq r, j \leqq s$. Now it must be observed that $w_{e}\left(x+a_{i}\right)$ $=w\left(a_{j}-a_{1}\right)$ or $w\left(a_{i}-b_{1}\right)$ for $i>r$, according to $r \neq 0$ or $s \neq 0$, and that similar fact holds for $b_{j}$.

1) When $r=n^{\prime}, s=m^{\prime}$ and $r \geqslant s$, we have obviously $w_{e}(d) \geqq w_{o}(d)$.

2) When $r<s$ and $r+s \neq m^{\prime}+n^{\prime}$ : Set $d^{\prime}=\Pi_{1}^{r}\left(x+a_{i}\right) / \Pi_{1}^{s}\left(x+b_{j}\right)$. Then $w_{e}\left(d^{\prime}\right)>w_{\sigma}\left(d^{\prime}\right)$ and therefore there exists on $b_{j}(j \leqq s)$ such that $w_{e}\left(d^{\prime}\right)$ $\geq w_{b_{j}}\left(d^{\prime}\right)$. Since the values of factors of $d$ other than those of $d^{\prime}$ are invariant under the replacement of $w_{e}$ by $w_{b_{j}}$, we have $w_{e}(d) \geqslant w_{b_{j}}(d)$.

3) When $r=n^{\prime}, s=m^{\prime}$ and $r<s$ : Let $\sigma^{*}$ be the minimum of values $w\left(a_{i}-a_{i^{\prime}}\right), w\left(a_{i}-b_{j}\right)$ and $w\left(b_{j}-b_{j}\right)$ and let $e *$ be an element of $K$ such that $w\left(a_{i}-e^{*}\right)=w\left(b_{j}-e^{*}\right)=\sigma^{*}$ for any $i, j^{2}{ }^{\prime} \quad$ Then since $w_{e}(d) \gtrsim w_{e^{*}}(d)$, we

Received May 22, 1955.

1) Prof. P. Ribenboim has communicated to the writer that the proof is not correct. The writer is grateful to him for his kind communication.

2) Such elements $e^{*}, e^{\prime \prime}$ and so on exist because $K$ is algebraically closed and therefore the residue class field of the valuation ring of $w$ is algebraically closed (and contains infinitely many elements). 
may replace $e$ by $e^{*}$. Next, let $\sigma^{* *}>\sigma^{*}$ be the next smallest value among $w\left(a_{i}-a_{i^{\prime}}\right), w\left(a_{i}-b_{j}\right)$ and $w\left(b_{j}-b_{j^{\prime}}\right)$ if they are not all equal; otherwise, we have obviously $w_{b_{j}}(d) \leqq w_{e}(d)$ for any $b_{j}$ and we have nothing to prove in this case.) We separate $a_{i}^{\prime} s$ and $b_{j}^{\prime} s$ to equivalent classes modulo the ideal of the valuation ring $\mathfrak{b}$ of $w$ generated by an element $e^{* *}$ of $K$ such that $w\left(e^{* *}\right)=\sigma^{* *}$. Since $r<s$, there exists a class $C=\left\{a_{i_{1}}, \ldots, a_{i_{t}}, b_{j_{1}}, \ldots, b_{j_{u}}\right\}$ such that $t<u$. Let $e^{\prime \prime}$ be an element of $K$ such that $w\left(a_{i_{l}}-e^{\prime \prime}\right)=w\left(b_{j_{l}}-e^{\prime \prime}\right)=\sigma^{* *}(k \leqq t, l$ $\leqq u)_{.}^{2)} \quad$ Then for other $a_{i}^{\prime} s, w\left(a_{i}-e^{\prime \prime}\right)=\sigma^{*}$; for other $b_{j}^{\prime} s, w\left(b_{j}-e^{\prime \prime}\right)=\sigma^{*}$. Hence we have $w_{e^{\prime \prime}}(d)<w_{e}(d)$. Applying the observation in 2) to $w_{e^{\prime \prime}}$, we have the required result.

4) Now we have only to treat the case when $r+s \neq m^{\prime}+n^{\prime}$ and $r \geqslant s$. Let $\sigma^{\prime \prime}$ be the maximum of values $w_{e}\left(x+a_{i}\right)(i>r)$ and $w_{e}\left(x+b_{j}\right)(j>s)$ and renumber $a_{i}$ and $b_{j}$ so that $w_{e}\left(x+a_{i}\right)=w_{e}\left(x+b_{j}\right)=\sigma^{\prime \prime}$ if and only if $r<i \leqq r^{\prime}, s \leqq j \leqq s^{\prime}$. Further let $e^{\prime}$ be an element of $K$ such that $w\left(a_{i}-e^{\prime}\right)$ $=w\left(b_{j}-e^{\prime}\right)=\sigma^{\prime \prime}$ for any $i \leqq r^{\prime}, j \leqq s^{\prime}{ }^{2)} \quad$ Since $r \geqq s$, we have $w_{e^{\prime}}(d) \leqq w_{e}(d)$ and we may replace $e$ by $e^{\prime}$. If we are still in the case 4$)$ with $w_{e^{\prime}}$, we repeat the similar process and we reach after a finite number of steps to one of the cases 1 ), 2), 3). Thus the lemma is proved completely.

Lemma B. Assume, in Lemma A, further that $m^{\prime} \geq n^{\prime}$ and $m^{\prime} \neq 0$. Then there exists one $b_{j}$ such that $w_{b j}(d)<w_{\sigma}(d)$.

Proof. Let $e$ be an element of $K$ such that $w(e)=w\left(a_{i}-e\right)=w\left(b_{j}-e\right)$ $=\sigma$ for any $i$ and $j^{2}$ ) Then we have $w_{e}(d)=w_{\sigma}(d)$. By virtue of Lemma A, we have only to show that there exists an element $e^{\prime \prime \prime}\left(w\left(e^{\prime \prime \prime}\right)=\sigma\right)$ such that $w_{e^{\prime \prime \prime}}(d)<w_{e}(d)$. If $m^{\prime}>n^{\prime}$, then by the same process in 3) above, we see the existence of $e^{\prime \prime \prime}$. Assume that $m^{\prime}=n^{\prime}$ and we will make use of induction argument on $m^{\prime}$. We apply the same process in 3) above. Then either there exists one class $C$ as above, which contains more $b_{j}^{\prime} s$ than $a_{i}^{\prime} s$, or any such classes have the same number of $a_{i}^{\prime}$ s and $b_{j}^{\prime}$ s. In the former case, take the element $e^{\prime \prime}$ as above (with respect to this class $C$ ). Then $w_{e^{\prime \prime}}(d)<w_{e}(d)$ and the assertion is proved in this case. On the other hand, let, say, $C=\left\{a_{i}, b_{i}\left(i \leqq r^{\prime \prime}\right)\right\}$ be an equivalent class in the latter case. Then since $r^{\prime \prime}<m^{\prime}$, we see the

3) If we take $\sigma^{* *}$, in this case, to be any number in $G$ which is greater than $\sigma^{*}$, then we see also the proof by the same way as below. 
existence of an element $e^{\prime \prime \prime}$ of $K$ sucht that $w_{e^{\prime \prime \prime}}\left(d^{\prime \prime}\right)<w_{e}\left(d^{\prime \prime}\right)$, where $d^{\prime \prime}=$ $\Pi_{1}^{r^{\prime \prime}}\left(x+a_{i}\right) / \Pi_{1}^{r^{\prime \prime}}\left(x+b_{i}\right)$. Since there exists one $b_{j}$ such that $w\left(b_{j}-e^{\prime \prime \prime}\right)$ is greater than some $w\left(a_{i}-e^{\prime \prime \prime}\right)\left(i, j \leqq r^{\prime \prime}\right)$, we see that $w\left(a_{i}, e^{\prime \prime \prime}\right)$ and $w\left(b_{j}\right.$, $\left.-e^{\prime \prime \prime}\right)$ are all equal for $i^{\prime}, j^{\prime}>r^{\prime \prime}$. Therefore we have $w_{e^{\prime \prime \prime}}(d)<w_{e}(d)$ and the assertion is proved.

Now we will return to the proof of the theorem.

First we assume that $w_{\lambda_{0}}(c)=0$ for some $\lambda_{0}\left(\alpha \leqq \lambda_{0} \leqq 2 \alpha\right)$. Let $i_{0}, r, j_{0}$ and $s$ be such that $w\left(a_{i}\right)=\lambda_{0}$ if and only if $i_{0}<i \leqq i_{0}+r, w\left(b_{j}\right)=\lambda_{0}$ if and only if $j_{0}<j \leqq j_{0}+s$. Set $\lambda_{1}=\max \left(\alpha, w\left(a_{i_{0}}\right), w\left(b_{j_{0}}\right)\right), \lambda_{2}=\min \left(2 \alpha, w\left(a_{i_{0+r+1}}\right), w\left(b_{j_{0+s+1}}\right)\right)$.

Then

$$
\begin{array}{r}
w_{\lambda_{1}}(c)=w\left(c_{0}\right)+\sum_{i \leqq i_{0}} w\left(a_{i}\right)-\sum_{j \leqq j_{0}} w\left(b_{j}\right)+\left(n-i_{0}\right) \lambda_{1}-\left(m-j_{0}\right) \lambda_{1} \geqq 0, \\
w_{\lambda_{0}}(c)=w\left(c_{0}\right)+\sum_{i \leqq i_{0}} w\left(a_{i}\right)-\sum_{j \leqq j_{0}} w\left(b_{j}\right)+\left(n-i_{0}\right) \lambda_{0}-\left(m-j_{0}\right) \lambda_{0}=0 \\
w_{\lambda_{2}}(c)=w\left(c_{0}\right)+\sum_{i \leqq i_{0}} w\left(a_{i}\right)-\sum_{j \leqq j_{0}} w\left(b_{j}\right)+r \lambda_{0}+\left(n-r-i_{0}\right) \lambda_{2}-s \lambda_{0} \\
-\left(m-s-j_{0}\right) \lambda_{1} \leqq 0 .
\end{array}
$$

Hence we have

$$
w_{\lambda_{1}}(c)=w_{\lambda_{1}}(c)-w_{\lambda_{0}}(c)=\left(n-i_{0}\right)\left(\lambda_{1}-\lambda_{0}\right)-\left(m-j_{0}\right)\left(\lambda_{1}-\lambda_{0}\right) \gtrsim 0 .
$$

Hence, if $\lambda_{0} \neq \alpha$, we have $\lambda_{1}<\lambda_{0}$ and $n-i_{0} \leqq m-j_{0}$.

Similarly we have

$$
w_{\lambda_{2}}(c)=w_{\lambda_{2}}(c)-w_{\lambda_{0}}(c)=\left(n-r-i_{0}\right)\left(\lambda_{2}-\lambda_{0}\right)-\left(m-s-j_{0}\right)\left(\lambda_{2}-\lambda_{0}\right) \geqq 0 .
$$

Hence, if $\lambda_{0} \neq 2 \alpha$, we have $n-r-i_{0} \geqslant m-s-j_{0}$. Thus in the case when $\lambda_{0}$ is equal to neither $\alpha$ nor $2 \alpha$, we first have $r \leqq s$. If $s \neq 0$, then Lemma B shows that there exists one $b_{j}\left(j_{0}<j \leqq j_{0}+s\right)$ such that $w_{\lambda_{0}}(c)>w_{b_{j}}(c)$, which is a contradiction. Hence $r=s=0$. Therefore we have further that $n-i_{0}=m-j_{0}$. In the case when $\lambda_{0}=\alpha$ or $\lambda_{0}=2 \alpha$, we see easily that $r=s=0$ and $n-i_{0}$ $=m-j_{0}$ because $\alpha \notin G$. If $\lambda_{1} \neq \alpha$, then there exists one $a_{i}$ or $b_{j}$ such that $w\left(a_{i}\right)$ or $w\left(b_{j}\right)$ is equal to $\lambda_{1}$, which is a contradiction because $w_{\lambda_{1}}(c)=0$ by the above equality. Hence $\lambda_{1}=\alpha$. Similarly we have $\lambda_{2}=2 \alpha$. From $\lambda_{1}=\alpha$, we have $i_{0}=j_{0}=0$, whence $m=n$; from $\lambda_{2}=2 \alpha$, we have $a_{i}=b_{j}=0$ for all

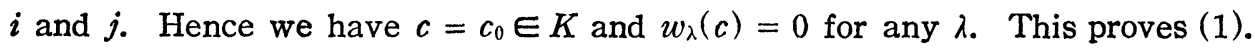
Next we assume that $w_{\alpha}(c)>0$. Let us consider $w_{\lambda}(c)$ as a function of variable $\lambda(\alpha \leqq \lambda \leqq 2 \alpha)$; it is obviously a continuous function and it takes the smallest 
and the largest values $\varepsilon_{1}$ and $\delta_{1}$ in $\alpha \leqq \lambda \leqq 2 \alpha$. By virtue of (1), we see that $\varepsilon_{1}$ is positive. Then (2) follows easily from the fact that $w_{e}(c) \neq w_{w(e)}(c)$ occurs only when $w(e)$ is one of $w\left(a_{i}\right)$ or $w\left(b_{j}\right)$; by the symmetricity of the assertion in Lemma $\mathrm{A}$, we see that these values $w_{e}(c)$ are bounded by the maximum and minimum of values $w_{w(e)}(c), w_{a_{i}}(c)$ and $w_{b_{j}}(c)$.

Since $w_{b_{j}}(c) \notin G$, the minimum is not zero and (2) is proved.

Mathematical Institute

Kyoto University 